Check for updates

1 Sheffield Teaching Hospitals NHS Trust

Cite this as: $B M J 2021 ; 375: n 3113$ http://dx.doi.org/10.1136/bmj.n3113 Published: 17 December 2021

\title{
Flattening the surgical field: ensuring diversity and maximising inclusion within surgical specialties
}

\section{Siri Gowda core surgical trainee}

Over the past few decades the argument for the need for diversification within the surgical field has been won. All specialties strive to deliver high quality care, and it has been repeatedly shown that marginalised groups are more likely to engage with healthcare services if they feel that they can identify with their physician. ${ }^{1}$ In recent times, the covid-19 pandemic has served as a stark reminder that racial and socioeconomic inequalities not only continue to exist, but remain pivotal in determining health outcomes. ${ }^{2}$

In order to reap tangible benefits from growing diversity, we need to ensure that minority groups feel accepted, and thereby able to contribute to change within departments. The route to true inclusivity lies in recognising and addressing existing barriers to entering surgery and progressing within the field, and in finding ways to widen the net when searching for future surgical talent.

Becoming a surgeon requires commitment to lengthy training, working long hours, and significant financial expenditure in the form of mandatory exams and courses. ${ }^{3}$ It is easy to understand why many young people do not even consider surgery as a viable career path.

As privilege is often already at play by the time young people enter university, interventions in schools in disadvantaged areas can help normalise surgery as a feasible career. The involvement of parents and guardians may help to initiate cultural change in underrepresented communities.

Mentorship initiatives can help address students' apprehensions about entering surgical training, and have been shown to improve the chances of candidates from underrepresented backgrounds securing higher surgical training posts, without diminishing competition ratios. ${ }^{1}$

Consideration must also be given to unconscious biases at play during recruitment. Skills-based standards help to avoid individual bias when recruiting, and the use of timely and accessible advertisements helps to prevent organisational factors from influencing who applies for certain posts. 4

Inclusivity is fundamental in retaining workforce diversity. This is particularly important in specialties that have higher attrition rates within underrepresented groups. Surgeons that have family and personal commitments, or religious beliefs may not be able to meet training curriculum demands in the same manner as those without. Additional support in the form of childcare, accommodation, and access to religious space within the working day, can enable people to remain within their specialties, and promote a culture of inclusivity. ${ }^{4}$
Ideally, barriers to accessibility should be pre-empted and resolved within trusts before trainees raising them as issues. As this is not always possible, surgical departments must ensure they regularly collect trainee feedback for equality monitoring. In order for people from disadvantaged groups to flourish and ascend to leadership positions they cannot be preoccupied with making their immediate surroundings and circumstances more amenable for themselves-this is an organisational responsibility.

Finally, all groups must feel like they belong within their sub-specialties; this often requires a cultural change. Guidance on how to address prejudice in surgery can be used as a framework to dismantle unhelpful stereotypes. The employment of equality and wellbeing committees can also increase a department's success in ensuring staff feel welcome. ${ }^{4}$

Improving inclusivity within the surgical profession mandates organisational and cultural change. While it is recognised that there is an increasing proportion of women and trainees from ethnic minority groups within surgery, less information is available regarding those with disabilities and socioeconomic disadvantage-implying more work needs to be done in these areas.

We must devise strategies to ensure marginalised groups are ascending to leadership positions with the same ease as their counterparts, and feel comfortable once they get there. Equal distance to the goalpost doesn't mean the game is fair if the playing field remains uneven.

Competing interests: none declared

Provenance and peer review: not commissioned, not peer reviewed

Butler, PD. Aarons, CB. Ahn, J. Wein, AJ. Ruckenstein, MJ. Lett, LA. DeMatteo, RP. Serletti, JM. Leading From the Front, Annals of Surgery: June 2019 Volume 269 - Issue 6 - p 1012-1015 doi: 10.1097/SLA.0000000000003197

2 Badran A, Mohamed W, Giwa L, Cheruvu MS. COVID-19, BAME and surgical training: a status quo? The Bulletin of the Royal College of Surgeons of England 2020;102(S1):4-8doi: 10.1308/rcsbull.TB2020.3.

3 O'Callaghan J, Mohan HM, Sharrock A, etalCouncil of the Association of Surgeons in Training. Cross-sectional study of the financial cost of training to the surgical trainee in the UK and Ireland. BMJ Open 2017;7:e018086. doi: 10.1136/bmjopen-2017-018086. pmid: 29146646

4 Royal College of Surgeons. Avoiding unconscious bias: a guide for surgeons. Published May 2016. https://www.rcseng.ac.uk/library-and-publications/rcspublications/docs/avoiding-unconscious-bias/ 